\title{
Conductas disruptivas en adolescentes en situación de deprivación sociocultural
}

\section{Disruptive behaviors in adolescents in a situation of sociocultural deprivation}

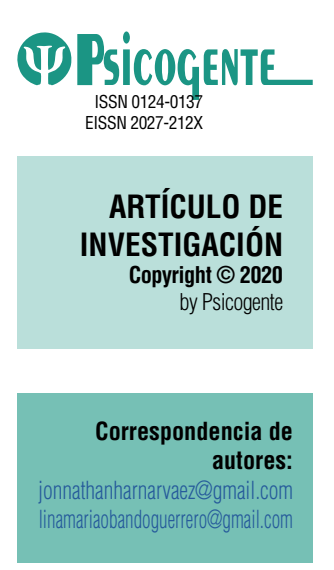

Recibido: 15-07-19 Aceptado: $02-02-20$ Publicado: 01-08-20

\author{
Jonnathan H. Narváez (iD) \\ Universidad de Nariño, Pasto, Colombia \\ Lina M. Obando iD \\ Universidad de Buenos Aires, Buenos Aires, Argentina
}

\section{RESUMEN}

Introducción: La deprivación sociocultural es un fenómeno multifactorial que incide en los procesos de desarrollo y adaptación de los individuos a sus contextos de socialización y aprendizaje. De ahí la importancia del estudio de los factores predisponentes de la deprivación sociocultural y la disrupción escolar, entre tanto brinda insumos interpretativos que favorecen la comprensión de la influencia de las dinámicas socioambientales en los procesos relacionales y de convivencia en la escuela.

Objetivo: Caracterizar las conductas disruptivas en adolescentes en situación de deprivación sociocultural favoreciendo un marco de comprensión que permita entender la génesis de los factores de disruptividad en las dinámicas interaccionales Escuela-Comunidad.

Método: A nivel metodológico se suscribió en el paradigma cualitativo con enfoque histórico-hermenéutico y método micro-etnográfico, se utilizaron técnicas como las entrevistas a profundidad y observación participante; el estudio contó con 80 estudiantes provenientes de las comunas 10, 6 y 3, 30 de género femenino y 50 de género masculino con rango de edad de los 13 a 17 años en la ciudad de Pasto, Nariño, con reportes de disrupción escolar y cuyos ambientes sociocomunitarios evidencian factores predisponentes a la deprivación sociocultural.

Resultados: Se logran identificar las conductas disruptivas que afectan el proceso de enseñanza aprendizaje y los factores de deprivación sociocultural asociados, seguidamente expone las conductas disruptivas con incidencia en los procesos de convivencia escolar, y se bosqueja un marco interpretativo sobre las consecuencias de la disruptividad en las dinámicas escolares.

Conclusiones: La deprivación sociocultural evidencia una situación de desventaja social al momento que los escolares expuestos a sus factores predisponentes cuentan con menos herramientas para la adaptación y aprovechamiento de la experiencia escolar, es además, uno de los detonantes de la disrupción escolar, fenómeno multifactorial que afecta tanto los procesos convivenciales como académicos; y un factor predictor del fracaso escolar.

Palabras clave: conductas disruptivas, deprivación sociocultural, ambiente escolar, escuela-comunidad.

\section{ABSTRACT}

Introduction: Sociocultural deprivation is a multifactorial phenomenon that affects the processes of development and adaptation of individuals to their contexts of socialization and learning. Hence, the importance of studying the predisposing factors of sociocultural deprivation and school disruption, meanwhile, it provides interpretative inputs that promote the understanding of the influence of socioenvironmental dynamics on the relationship and coexistence processes in the school.

Objective: To characterize the disruptive behaviors in adolescents in a situation of sociocultural deprivation, favoring a framework of understanding that allows us to understand the genesis of disruptive factors in the interaction dynamics of the School-Community.

Method: At the methodological level, the qualitative paradigm was subscribed with a historical-hermeneutical approach and a micro-ethnographic method, using techniques such as in-depth interviews and participant observation; The study included 80 students from communes 10, 6, and 3, 30 female and 
50 male, with an age range of 13 to 17 years in the Pasto city, with reports of school disruption and whose socio-community environments show predisposing factors to the sociocultural deprivation.

Results: It is possible to identify the disruptive behaviors that affect the teaching-learning process and the associated sociocultural deprivation factors, then it exposes the disruptive behaviors with incidence in the processes of school coexistence, and outlines an interpretative framework on the consequences of the disruption in school dynamics.

Conclusions: Sociocultural deprivation shows a situation of social disadvantage at the time that schoolchildren exposed to its predisposing factors have fewer tools for adapting and taking advantage of school experience, it is also one of the triggers of school disruption, a multifactorial phenomenon that affects both coexistence and academic processes; and a predictor of school failure.

Keywords: disruptive behaviors, sociocultural deprivation, school environment, school- community.

\section{INTRODUCCIÓN}

Las prácticas educativas han sufrido diferentes transformaciones como influjo de los factores socioambientales y de los cambios acelerados que se derivan de la globalización, sumada a fenómenos contemporáneos comunes a diversos escenarios sociopolíticos de América Latina como la desigualdad, la violencia y el crecimiento urbano a gran escala. De acuerdo a Peña y Angulo (2013) estos fenómenos inciden de manera directa sobre los establecimientos educativos, por cuanto acogen a escolares provenientes de diversos ambientes sociales que no ofrecen las condiciones óptimas para el proceso educativo y el desarrollo humano; en consecuencia, como señala Bosch (2003) la escuela puede considerarse una institución en permanente crisis, por cuanto al orientar el proceso de socialización de los seres humanos queda a la zaga de las necesidades sociales del momento.

Es así, que los establecimientos educativos al estar inmersos en ambientes socio-culturales heredan de los mismos una serie de dinámicas y prácticas, las cuales inciden de manera sustantiva en el cumplimiento de los objetivos educacionales, afectando el rendimiento académico y el ambiente escolar. Uno de los fenómenos de mayor incidencia en las escuelas es la deprivación sociocultural, la cual es definida como una capacidad reducida que tienen los individuos "para modificar sus estructuras intelectuales y responder a las fuentes externas de estimulación" (Cedillo, 2010, p. 29), o considerada como un conjunto de circunstancias que van a impedir o dificultar el "normal desarrollo cognitivo, físico, emocional y/o social de las personas que viven inmersas en ambientes de pobreza cultural y/o material" (Jiménez, 2009, p.1), lo que representa una carencia en la transmisión cultural por razones internas o por las condiciones ambientales en las que se ve inmerso el sujeto, lo que constituye un estado de desventaja observable y demostrable en la falta de contacto social (Barello, Palmucci \& Schuager, 2007, citados en Fuica, et al., 2014), que puede relacionarse con diversas dificultades en el 
área personal o grupal de un sujeto, llegando a afectar aspectos básicos de su vida cotidiana, entre ellos la adaptación al contexto escolar, los procesos de socialización, la comunicación interpersonal e intrapersonal así como el rendimiento académico.

Entre la caracterización de los escenarios predisponentes a situaciones de deprivación sociocultural, López y Encabo (2000) plantean que existe un mayor riesgo en aquellas personas que viven en situación de precariedad económica y aquellas cuyas redes de apoyo son inoperantes, sin embargo, frente a lo primero Narváez (2019) aclara que si es cierto, que la privación material es un factor de riesgo a la deprivación sociocultural no es su factor constituyente, en la medida, que la deficiente transmisión cultural tiene como factor fundamental la calidad de vínculos que establece un individuo con su entorno más allá de la pobreza material en la que esté inmerso, tal afirmación explica las diferencias individuales que pueden presentar estudiantes que provienen de los mismos contextos de privación material y presentan un buen nivel de desarrollo cognitivo, destacado desempeño personal y social, así como un nivel de aprovechamiento global superior. Al respecto, Herrera (2000) subraya entre otros factores de deprivación, el inadecuado ambiente familiar, bajo nivel escolar, cultural y económico de los cuidadores, ambientes comunitarios violentos y rasgos antisociales en los grupos de influencia.

Tanto las condiciones socioculturales, es decir, el contexto comunitario y escolar, como la deprivación sociocultural, se encuentran íntimamente ligados a los procesos educativos, entre tanto es función de la escuela el proveer herramientas que permitan subvertir los efectos de los ambientes predisponentes a la deprivación sobre el desarrollo humano de los individuos (Narváez, 2019). De manera, que los establecimientos educativos enfrentan fenómenos derivados de la deprivación como la desmotivación, poca adaptabilidad al entorno educativo, fracaso escolar y los elevados índices de disruptividad (desobediencia, incumplimiento de normas, acciones que interrumpen el ritmo de la clase como: ruidos, chistes, burlas, gritos, golpes e insultos); manifestaciones que interfieren en la labor educativa de los maestros y evidencian algunas de las señales de la deprivación sociocultural sobre las relaciones interpersonales y el clima escolar.

La disrupción escolar se entiende como aquellas conductas mediante las cuales los estudiantes interrumpen el normal funcionamiento de una clase por constituir transgresiones a normas establecidas en la institución (Gordillo, 2013), en otras palabras, sucede cuando los comportamientos de los alumnos no encajan con los valores, motivaciones $u$ objetivos del proceso educativo 
(Díaz, 2012). Por su parte, Álvarez, Castro, González, Álvarez y Campo (2016), plantean que las conductas disruptivas se tipifican por el incumplimiento de normas y acuerdos que conducen a lo que generalmente se denomina mal comportamiento, agresividad, molestias reiteradas, indisciplina, groserías, transgresión de la verdad, déficit de atención, falta de cooperación, desobediencia, impertinencia y amenazas. De igual modo, Pino y García (2007 citado por Sulbaran y León, 2014), señalan que las conductas disruptivas suelen ser hechos violentos, en los que tiene lugar la agresión a un profesor o algún suceso similar entre alumnos, así como los conflictos, la impuntualidad, burlarse del docente, falta de respeto, agresiones verbales y físicas. Estas conductas, poco a poco, van incidiendo en el quehacer del docente, contribuyendo a minar o afectar su moral y motivación.

En la misma línea, Ruiz, Riuró y Tesouro (2015) sugieren que un tipo de conductas disruptivas es el maltrato verbal el cual se produce a través de palabras, apodos, burlas, insultos, mentiras, difusión de rumores de la víctima, críticas a sus espaldas, entre otros; mientras Huertas (2013), añade que es violencia verbal todo lo relacionado con bloquear y desviar, acusar y culpar, juzgar y criticar, trivializar, destruir, amenazar, insultar, negar y manifestar ira abusiva en contra de algún compañero, lo que da cuenta de carencia de herramientas para el autocontrol, uno de las manifestaciones personales de un sujeto socialmente deprivado. A esto se suma comúnmente la agresión física la cual hace referencia a un conjunto de conductas que se realizan con el fín de generar un daño físico y psicológico a la persona que es víctima, comportamiento característico de entornos familiares y comunitarios socialmente violentos, tendientes a la deprivación.

Sin duda, tanto la deprivación sociocultural como las conductas disruptivas influyen en el proceso de enseñanza-aprendizaje, dado que el aula se convierte en un espacio donde sus manifestaciones llevan a que los docentes empleen más tiempo en el control de la misma que en fomentar y estimular el desarrollo cognitivo (Sulbaran y León, 2014); situación que altera el rendimiento escolar, la planificación académica y disminuye los niveles de desempeño, minimizando el efecto de la escolarización en el afrontamiento efectivo de ciertos factores socioambientales que menguan los procesos de socialización y desarrollo humano (Cladellas, Clariana y Badia, 2015), por lo anterior, Sulbaran y León (2014) concluyen que las conductas disruptivas son incompatibles con los procesos de aprendizaje y repercuten en la convivencia escolar, bloqueando el carácter emancipador de la relaciones pedagógicas como potenciales fuentes de estimulación que permitan minimizar el impacto de los factores predisponentes a la deprivación. 
Sin embargo, no existe un acervo investigativo que relacione el fenómeno de la deprivación sociocultural y las conductas disruptivas, siendo uno de los propósitos de este artículo presentar un esbozo comprensivo donde se integran las dinámicas ambientales con los procesos convivenciales intra-aula. Al respecto, uno de los puntos de encuentro podría ser el estudio de las manifestaciones de deprivación escolar, en las cuales, Narváez (2019) manifiesta que la calidad en los vínculos del maestro (en función de mediador) y de sus estudiantes, favorece o minimiza los niveles de deprivación sociocultural, presentando como uno de los factores que llevan a la deprivación escolar al ambiente de aula, el cual es afectado sustancialmente por las conductas disruptivas las cuales suponen una ruptura en las dinámicas relacionales docente-estudiante, entre tanto, para garantizar la autoridad ante comportamientos sistemáticos de indisciplina los docentes en algunas ocasiones tienden a desarrollar reacciones agresivas (gritos), cometen acciones intimidatorias (levantamiento de actas, citaciones, bajar calificaciones), emiten castigos repetitivos los cuales desencadenan episodios de conflicto entre los miembros de la institución (Peña y Angulo, 2013); situaciones que debilitan la calidad de los vínculos y la influencia positiva del maestro en la formación de los estudiantes. Estos conflictos conllevan a que la institución pase de ser un ambiente pedagógico abierto a uno hostil, menguando el potencial mediador del maestro en el proceso educativo.

Tal situación se presenta en diversos centros escolares cuyos estudiantes provienen de contextos con factores predisponentes a la deprivación sociocultural, tales como, escenarios comunitarios permeados por la violencia urbana, familias con relaciones de disfuncionalidad y precariedad económica, nichos barriales con deficientes redes de apoyo y soporte social, o distanciamiento afectivo en las familias. En efecto, el presente artículo tiene como intención ofrecer un marco interpretativo entre los factores predisponentes a la deprivación sociocultural y la disrupción escolar, de modo que pueda comprenderse la influencia de las dinámicas socioambientales en los procesos relacionales y de convivencia en la escuela.

\section{MÉTODO}

\subsection{Diseño}

El estudio se enmarca en el paradigma cualitativo el cual busca comprender la posición, interpretación y el significado que los sujetos construyen a partir de la significación de sus experiencias (Castaño y Quecedo, 2002). De este modo, se propende por la comprensión de los fenómenos de la deprivación sociocultural y las conductas disruptivas como dinámicas emergentes del 
ambiente escolar a partir del develamiento de actitudes, pensamientos e interpretaciones que los sujetos hacen de esta realidad, en la inmersión de los investigadores al contexto (Hernández, Fernández y Baptista, 2010).

A su vez, la investigación se desarrolla con base al enfoque histórico-hermenéutico, el cual mediante procesos libres y no estructurados favorece la identificación de las interpretaciones y construcciones de la realidad que el individuo e investigador vive y construye en un contexto concreto a partir de la indagación de los fenómenos desde marcos de análisis históricos (Delgado, 2010). Así, al delimitar las manifestaciones de deprivación sociocultural y las conductas disruptivas que se dan en el aula se integran ejes de análisis interpretativos que ofrecieron desde la indagación de la historia personal y los marcos relacionales de los participantes, una lectura integrativa de los fenómenos socioambientales y las dinámicas de aula.

Por su parte, se utilizó el método de la microetnografía o etnografía constitutiva pues existió el interés de profundizar en el análisis de los patrones con los que interactúan los miembros de una comunidad, haciendo énfasis en marcos interaccionales. Desde esta perspectiva, mediante este método se asume que la comunicación verbal y no verbal son culturalmente modeladas, aunque las personas en interacción no tengan conciencia de ello (Álvarez, 2008), premisa que es afín con los postulados de la deprivación sociocultural desde la relación sujeto-entorno y las conductas disruptivas como manifestaciones comunicativas de las interacciones socioculturales que los participantes aprenden y recrean al interior de la escuela. De igual forma, como plantea Serra (2004), la virtud de realizar estudios microetnográficos en escenarios escolares radica en su potencialidad para ofrecer nuevos datos, nuevas miradas y nuevas perspectivas que contribuyan a mejorar las propias prácticas educativas.

\subsection{Población y muestra}

80 Estudiantes provenientes de las comunas 10,6 y 3 de Pasto, 30 de género femenino y 50 de género masculino con rango de edad de los 13 a 17 años, los cuales integran los grupos que más reportes de disrupción escolar presentan en el establecimiento educativo, asimismo, presentan factores predisponentes a la deprivación sociocultural y cuentan con matricula regular en la IEM San Juan Bosco, Pasto, en el año 2018. Entre los factores predisponentes a la deprivación sociocultural se determinaron: a) residencia en ambientes comunitarios que presentan dinámicas de violencia urbana, b) dificultades en el proceso de adaptación escolar, c) reportes escolares de familias con 
disfuncionalidad. Por comuna se entiende a la unidad político administrativa, de la ciudad de Pasto, que integra un conjunto de barrios que comparten el entorno geográfico y sociopolítico.

\subsection{Instrumentos}

Para la recolección de la información se utilizaron dos instrumentos correspondientes a la observación participativa y la entrevista a profundidad. La observación y la observación participante proporcionan descripciones del fenómeno estudiado (Álvarez, 2008) desde una dinámica interactiva de los sujetos de estudio y el investigador. En primera medida se utilizó la observación participativa, la cual se constituye como una técnica de inspección y estudio de las cosas o hechos tal como acontecen en la realidad mediante el empleo de los sentidos, conforme a las exigencias de la investigación científica y a partir de las categorías emergentes que van ocurriendo (Yuni y Urbano, 2006, citados en Artigas y Robles, 2010); dicho ejercicio se realiza con el establecimiento previo de unos objetivos claros y precisos (Calvache, 2009). El objetivo de la aplicación de la técnica estuvo orientado a la delimitación de los diversos comportamientos disruptivos dentro de las aulas en un ambiente natural; entre los criterios de observación estuvieron: a) identificar las conductas disruptivas que afectaban el proceso de enseñanza aprendizaje, b) determinar la incidencia de las conductas disruptivas en la convivencia escolar, c) reconocer algunas consecuencias de la disruptividad en el proceso educativo.

El segundo instrumento que se utilizó fue la entrevista a profundidad, su relevancia radica en que "tejida sobre el diálogo, proporciona discurso ajeno, de los sujetos de estudio" (Velasco y Díaz de Rada, 2006 citado por Álvarez, 2008). Es decir, se basa en un diálogo cotidiano donde se evita la formalidad y se brinda la visión que los sujetos tienen de la realidad. Para una efectiva ejecución fue necesario propiciar un entorno de confianza, curiosidad y naturalidad, delimitando los informantes claves que propiciaron la mayor información posible. El objetivo de la aplicación estuvo orientado a conocer los factores predisponentes de carácter comunitario, familiar y escolar que dan cuenta de las manifestaciones de la deprivación sociocultural en la escuela.

\subsection{Procedimiento}

De acuerdo a Bojacá (2005), el estudio se orientó por tres etapas: a) la primera denominada fenomenológica, donde los investigadores posterior a su inserción en el ambiente educativo, se familiarizaron con el sujeto y 
fenómenos estudiados, esta fase consistió en el reconocimiento temático y contextual de la deprivación sociocultural y la disrupción en el aula desde una aproximación teórica y cotidiana, mediante registros de observación participativa de la experiencia pedagógica en el establecimiento educativo y la revisión documental; b) seguidamente, se da una etapa interpretativa, donde tanto investigador como sujetos de estudio, a partir de la entrevista a profundidad favorecen el reconocimiento de las vivencias individuales y colectivas relacionadas al fenómeno en estudio, situación que permitió la identificación de casos y la formulación de hipótesis analíticas; c) una vez concluida la interpretación de vivencias asociadas a los factores predisponentes a la deprivación sociocultural y los comportamientos disruptivos se establecieron categorías inductivas, las cuales reflejan y describen los hallazgos del estudio a través del cual se ofrece un acervo interpretativo de la realidad socio-educativa, de las prácticas y fenómenos en estudio, dando lugar al informe investigativo.

\subsection{Análisis de datos}

En coherencia con la naturaleza inductiva de los datos microetnográficos, el análisis de los mismos se dio en la aplicación de la estrategia de inducción analítica, la cual es un procedimiento para verificar, contrastar y generar teorías con base a proposiciones emergentes de las experiencias y significaciones subjetivas de los grupos con quienes se investiga. Para el análisis e interpretación de resultados el estudio siguió las etapas propuestas por Kharicha, Iliffe y Myerson (2013) y por Amezcua y Gálvez Toro (2002) quienes plantean que, en la primera etapa, se deben definir los fenómenos a investigar a partir de la revisión bibliográfica y de antecedentes. Lo anterior con la finalidad de establecer una definición aproximada de los fenómenos de disrupción escolar y de deprivación sociocultural.

La segunda etapa consistió en la formulación de hipótesis que podrían explicar el fenómeno u objetivo a investigar, para así proceder a la siguiente fase que se caracteriza por la identificación de casos que permitieran verificar si las hipótesis se ajustan a la realidad educativa estudiada. Para los casos donde las hipótesis no explicaban el fenómeno, se derivó a la reformulación de las hipótesis. Posteriormente, se llevó a cabo la búsqueda activa de negativos que refuten las hipótesis, lo cual se repitió hasta que la información y el marco explicativo fueran congruentes. Una vez alcanzada la congruencia integrativa entre los datos y las hipótesis se dio por terminada la fase de resultados. Para 
finalizar el plan de análisis, se procedió a la triangulación de las hipótesis y casos aceptados, en aras de establecer el informe interpretativo del estudio.

\section{RESULTADOS}

La disrupción en las aulas constituye una de las preocupaciones más directas y una de las principales fuentes de malestar en los diversos Centros Educativos, tal es el caso de la Institución Educativa Municipal San Juan Bosco en Pasto, Colombia, donde se presentan de manera sistemática comportamientos que afectan tanto el proceso de enseñanza-aprendizaje como las dinámicas convivenciales.

\subsection{Conductas disruptivas que afectan el proceso de enseñanza aprendizaje}

Desde el marco de interpretación de la vida escolar de los estudiantes que presentaban predisposición ambiental a la deprivación sociocultural, se develaron marcos interaccionales que dan cuenta de la práctica de comportamientos disruptivos; los cuales para la presentación de resultados se clasifican en aquellos que afectan el proceso de enseñanza-aprendizaje, divididos a su vez, en los relacionados con el rendimiento y desempeño académico, es decir, todas aquellas conductas asociadas al no aprovechamiento didáctico, el incumplimiento de objetivos escolares, así como al óptimo desempeño de los estudiantes en diversas actividades de construcción de aprendizaje o evaluación, las tres, condiciones relevantes para garantizar escenarios de enseñanza satisfactorios.

De igual manera, se presentan conductas relacionadas a la interrupción de la clase, donde se integra el conjunto de comportamientos que obstaculizan el aprovechamiento efectivo del tiempo y los procesos atencionales del grupo, siendo focos de distracción e indisciplina dentro del aula; también se presenta absentismo, condición fundamental para identificar los aspectos motivacionales, de responsabilidad y autogestión formativa, por cuanto es evidente, la sistemática inasistencia escolar o retardos a clase; finalmente, encontramos conductas disruptivas en relación a la presentación personal y el cuidado del medioambiente educativo, siendo característico el porte inadecuado del uniforme y el daño intencional de bienes, muebles y de infraestructura en aquellos grados donde más se presenta disrupción. 
La Tabla 1 presenta los comportamientos disruptivos que afectan al proceso de enseñanza-aprendizaje y su relación con algunos factores de deprivación sociocultural.

TABLA 1.

Conductas disruptivas que afectan al proceso de enseñanza-aprendizaje

\begin{tabular}{|c|c|c|}
\hline \multicolumn{3}{|c|}{ CONDUCTAS DISRUPTIVAS QUE AFECTAN AL PROCESO DE ENSEÑANZA-APRENDIZAJE } \\
\hline $\begin{array}{l}\text { CLASIFICACIÓN DE LAS } \\
\text { CONDUCTAS }\end{array}$ & COMPORTAMIENTOS DISRUPTIVOS & $\begin{array}{l}\text { FACTORES PREDISPONENTES DE DEPRI- } \\
\text { VACIÓN SOCIOCULTURAL ASOCIADOS }\end{array}$ \\
\hline $\begin{array}{l}\text { Relacionadas con el rendimiento y } \\
\text { desempeño académico }\end{array}$ & $\begin{array}{l}\text { - No realización de tareas en el hogar. } \\
\text { - } \quad \text { Falta de interés, pasividad e inactividad en clase. } \\
\text { el buentar con losarrollo de la clase (copias, esferos, } \\
\text { cuadernos...). } \\
\text { - Lentitud en el trabajo. } \\
\text { - Realizar dentro de una clase actividades de otras } \\
\text { - } \text { asignaturas. } \\
\text { - } \quad \text { Cosistencia frente a la asignación de tareas en clase. } \\
\text { exámenes. }\end{array}$ & $\begin{array}{l}\text { Deprivación familiar } \\
\text { - } \quad \text { Bajo nivel educativo de los padres. } \\
\text { - } \text { mentarcia de apoyo emocional e instru- } \\
\text { - } \quad \text { Precario nivel socioeconómico. } \\
\text { - Escasa proyección y expectativas futuras } \\
\text { en la familia. }\end{array}$ \\
\hline Interrupción de la clase & $\begin{array}{l}\text { - Interrupciones dirigidas a obstaculizar el desarrollo } \\
\text { - } \text { de contenidos. } \\
\text { - Sacer ruidos o gritar en clase. } \\
\text { - Levantarse del sitio sin permiso. } \\
\text { - Hablar continuamente en clase. } \\
\text { - Ingerir alimentos en clase. } \\
\text { - Jugar dentro del salón de clase o en actividades de } \\
\text { - } \quad \text { formación conjunta. } \\
\text { - Uscuchar música. } \\
\text { - Tirar papecuado de medios electrónicos. }\end{array}$ & $\begin{array}{l}\text { Deprivación escolar } \\
\text { - Estigmatización por parte de los } \\
\text { docentes. } \\
\text { - Carencia de medios e infraestructura } \\
\text { educativa. } \\
\text { - Permisividad normativa. }\end{array}$ \\
\hline Absentismo & $\begin{array}{l}\text { - Faltar a clase. } \\
\text { - Llegar tarde a clase. } \\
\text { - Retrasos sin justificar. } \\
\text { - Retraso en el ingreso al curso y en el inicio de } \\
\text { clases. }\end{array}$ & $\begin{array}{l}\text { - Nivel socioeconómico del grupo } \\
\text { famililar. } \\
\text { - Desmotivación y bajas expectativas } \\
\text { escolares. } \\
\text { - Permisividad y falencias en las figuras } \\
\text { de autoridad parentales. }\end{array}$ \\
\hline $\begin{array}{l}\text { Presentación personal y cuidado } \\
\text { del medioambiente educativo }\end{array}$ & $\begin{array}{l}\text { - Consumo de psicoactivos. } \\
\text { - Rayar las mesas o paredes. } \\
\text { - Portar el uniforme de manera inapropiada. } \\
\text { - Uso permanente de accesorios restringidos en el } \\
\text { establecimiento. }\end{array}$ & $\begin{array}{l}\text { Deprivación comunitaria } \\
\text { - Aprendizaje y reproducción de compor- } \\
\text { tamientos antisociales. } \\
\text { - Normalización de conductas violentas } \\
\text { - Relativización de las normas. }\end{array}$ \\
\hline
\end{tabular}

\subsection{Conductas disruptivas que inciden sobre la convivencia escolar}

Entre estas encontramos las faltas de respeto que oscilan desde el desconocimiento de la autoridad al uso de una comunicación y lenguaje inadecuados, así como la no responsabilización frente a faltas personales; posteriormente, se ubican las conductas que implican conflictos de poder, donde se describen una serie de comportamientos desafiantes desde el uso de la violencia simbólica, la confrontación directa con figuras de autoridad y la territorialización de espacios físicos dentro y fuera del aula; finalmente, se 
señalan conductas relacionadas con situaciones de violencia que implican la afectación de la integridad física, psicológica y moral de bienes y actores del contexto educativo, destacándose que en el escenario escolar solo se presenta violencia física entre estudiantes. La Tabla 2 identifica los comportamientos disruptivos que inciden sobre la convivencia escolar.

Tabla 2.

Conductas disruptivas que inciden sobre la convivencia escolar

\begin{tabular}{ll}
\hline \multicolumn{2}{c}{ CONDUCTAS DISRUPTIVAS QUE INCIDEN SOBRE LA CONVIVENCIA ESCOLAR } \\
\hline CLASIFICACIÓN DE LAS CONDUCTAS & \multicolumn{1}{c}{ COMPORTAMIENTOS DISRUPTIVOS } \\
\hline & - Hablar cuando el profesor habla \\
& - No atender los llamados de atención y órdenes \\
Faltas de respeto & - Culpabilizar a otros estudiantes de faltas personales \\
& - Insultos \\
& - Burlas sistemáticas \\
& - Intanería \\
Conflictos de poder & - Confrontación y amenazas directa con el profesor. \\
& - Sectorización de ambientes y espacios escolares \\
& - Desafíos de la autoridad \\
Situaciones de violencia & - Daño intencional de materiales educativos de los compañeros \\
& - Exclusión verbal, física o simbólica \\
\hline
\end{tabular}

El ambiente educativo dinamiza una serie de factores de deprivación sociocultural en los niveles sociofamiliar y ambiental, que evidencian comportamientos que afectan el proceso de enseñanza-aprendizaje y la convivencia dentro del centro educativo. Entre los principales factores encontramos tres tipos: a) Relacionados con las actividades académicas, b) Relacionados a la interacción entre estudiantes, y c) Relacionados con el uso adecuado de la planta física, medioambiente y equipos.

En relación a las actividades académicas se presenta evasión de clase, impuntualidad en el cambio de hora o después del descanso, ausencia de normas, actitud poco colaborativa y resistente ante las actividades de enseñanza, indisciplina en clase y poca autodidaxia formativa. Por su parte, en materia de interacción entre pares, se presentan situaciones de agresión verbal permanente, cutting, porte de prendas ajenas al uniforme, o uniforme incompleto, uso de términos ofensivos y morbosos en la comunicación interpersonal, manejo de lenguaje corporal y gestual agresivo, apodos, práctica de juegos bruscos, discriminación y exclusión por sexo, condición física, etnia e identidad sexual, manejo de roles de poder abusivos, conflictos interpersonales, consumo de sustancias psicoactivas, acoso escolar y ciberacoso. En cuanto a las situaciones relacionadas con el uso de la planta física, el 
medioambiente y los equipos, existe manejo inadecuado de basuras, uso inapropiado del volumen de voz, hurtos, rayones en muebles, daños en plantas y zonas comunes, deterioro de chapas, uso indebido de los sanitarios e inadecuada higiene personal.

\subsection{Consecuencias de la disruptividad escolar en las dinámicas escolares}

Finalmente, se propusieron hipótesis inductivas en relación a las causas y consecuencias de las conductas disruptivas en entorno escolar. Entre las causas que explican las conductas disruptivas de los estudiantes en relación con los factores socioambientales y escolares encontramos indicadores familiares y comunitarios de deprivación sociocultural, como lo son los patrones sociales de violencia que son reproducidos desde sus nichos comunitarios, los cuales heredan comportamientos agresivos y violentos, pérdida de la autoridad moral de los adultos, y falta de reconocimiento de la importancia de la educación y de los objetivos educativos, inversión de roles y sistemas normativos permisivos, diferencias etarias entre el alumnado, y escolarización punitiva y obligatoria por parte de los padres de familia. Finalmente, la carencia de herramientas de afrontamiento de los comportamientos disruptivos por parte de algunos profesores. En cuanto a las consecuencias, la Tabla 3, las clasifica de acuerdo a diversos actores del ambiente educativo, agentes de disrupción escolar, docentes y directivos docentes, compañeros de clase y padres de familia.

Tabla 3.

Consecuencias de la disruptividad escolar

\begin{tabular}{|c|c|}
\hline \multicolumn{2}{|r|}{ CONSECUENCIAS DE LA DISRUPTIVIDAD ESCOLAR } \\
\hline ACTOR & CONSECUENCIA \\
\hline \multirow{5}{*}{ Agentes de disrupción escolar } & - Rendimiento académico bajo. \\
\hline & - Poca adaptación al entorno escolar y normativo. \\
\hline & - Fracaso escolar. \\
\hline & - Deserción educativa. \\
\hline & - Reproducción de comportamientos desadaptativos. \\
\hline \multirow{4}{*}{ Docentes y Directivo docentes } & - Saturación en procesos de convivencia. \\
\hline & - Estrés laboral. \\
\hline & - Dificultad en la creación de estrategias de enseñanza y evaluación. \\
\hline & • Tensión y desmotivación. \\
\hline \multirow{5}{*}{ Compañeros de clase } & $\begin{array}{l}\text { - Influye negativamente en el aprendizaje y el rendimiento escolar de todos } \\
\text { los estudiantes. }\end{array}$ \\
\hline & - Genera un clima de aula inadecuado. \\
\hline & $\begin{array}{l}\text { - Impide el buen desarrollo de los procesos de enseñanza-aprendizaje. } \\
\text { - Estrés académico. }\end{array}$ \\
\hline & - Tensión y desmotivación. \\
\hline & - Confrontación y conflicto. \\
\hline \multirow{3}{*}{ Padres de familia } & - Asistencia frecuente a la institución por los llamados de los directivos. \\
\hline & $\begin{array}{l}\text { - Controntación padres e hijos. } \\
\text { - Pérdida de la autoridad parental. }\end{array}$ \\
\hline & - Choques y confrontaciones entre los cuidadores. \\
\hline
\end{tabular}




\section{DISCUSIÓN}

La finalidad del presente estudio fue caracterizar las conductas disruptivas en adolescentes con factores predisponentes de deprivación sociocultural. Al respecto Montoya, Giraldo, Arango, Forgiarini y García (2014) argumentan, que la precariedad económica y algunas características de los padres o familia como el bajo nivel educativo o la ausencia de estrategias educativas genera dificultades en los procesos cognitivos de los sujetos, quienes pueden presentar déficit en el razonamiento no verbal y espacial, alteración de los procesos atencionales, poca eficiencia en la realización de tareas, entre otras, lo que se traduce en la generación de comportamientos disruptivos; postulados que se verifican en los hallazgos del estudio, entre tanto se encuentra un marco de relación entre factores como la carencia de apoyo emocional o el precario nivel socioeconómico de las familias, con comportamientos disruptivos que afectan el rendimiento y desempeño académico.

En relación a los factores predisponentes a la deprivación en el ambiente familiar, en consonancia a lo propuesto por Andrade, Gonzales y Calle (2019), quienes establecieron que una relación sana con los padres mejora sus habilidades para la vida, pero disminuye el reconocimiento de riesgos en el hogar y el ambiente; los adolescentes que son expuestos a dinámicas de deprivación familiar, como la carencia de apoyo emocional e instrumental o la escasa proyección a expectativas futuras en la familia, tienden a desarrollar comportamientos disruptivos como la no realización de tareas en el hogar, falta de interés y absentismo, así como niveles de conflictividad e indisciplina creciente en el aula. Todas ellas, evidentes manifestaciones de la deficiencia en las habilidades para la vida, situación que acarrea consecuencias negativas tanto en los procesos convivenciales como académicos del individuo y la escuela.

Entre las consecuencias de la disruptividad escolar en los agentes de disrupción, el estudio denota la baja adaptación al entorno escolar y normativo y el rendimiento académico bajo, situaciones que derivan en la sistemática confrontación entre padres e hijos, escenario que dificulta el establecimiento de roles y el adecuado clima familiar, marco relacional que obstaculiza aún más el desempeño académico interpelando la funcionalidad parental. Lo anterior, se corrobora con lo propuesto por Lastre, López y Alcázar (2018), quienes al establecer la relación entre el apoyo familiar y el rendimiento académico de los estudiantes, lograron determinar que las variables tienen una relación estadísticamente significativa por lo que se puede plantear que aquellos padres que acompañan, retroalimentan y están pendientes de la 
vida escolar, sus hijos muestran mejores niveles de desempeño escolar, o viceversa, como es evidente en quienes son expuestos a factores de deprivación familiar.

Benavides, Muñoz y Muñoz (2013) añaden que el aprendizaje es un proceso social, de modo que el conjunto de interacciones ambientales determina ciertos patrones adaptativos y comportamentales, razón por la cual se justifica que los sujetos que tiendan a la privación material o deprivación sociocultural puedan presentar conductas disruptivas o dificultades en la adaptación a los contextos escolares. En este escenario, Moreno (2017) propone que una de las manifestaciones más frecuentes en los ambientes educativos es la violencia verbal, tal como puede evidenciarse en los comportamientos disruptivos del sujeto de estudio, y agrega que dichas conductas tienden a ser naturalizadas como consecuencia de su recurrente reproducción en los ambientes escolar, familiar y comunitario. Lo anterior, da cuenta de la relación existente entre las condiciones del ambiente societal en donde están inmersos los escolares y el tipo de comportamiento que estos tienen dentro de las aulas, de allí que podría inferirse que a mayores factores predisponentes a la deprivación sociocultural mayor prevalencia de comportamientos disruptivos (Narváez, 2019).

Situación que se contrasta con lo propuesto por Carrillo, Civis, Blanch, Longás y Riera (2018) cuando analizan los condicionantes de éxito y fracaso escolar en estudiantes en contextos de bajo nivel socioeconómico, donde determinan que un factor trasversal al fracaso escolar está relacionado con la precariedad de vínculos sociales entre los agentes socializadores y los alumnos, situación que favorece o no la consolidación de unos recursos internos que permitan el afrontamiento efectivo de la vida escolar y una adaptación satisfactoria a la escuela. De manera, que los comportamientos disruptivos implican no solo deficiencias en la adaptación simbólica al aula y los procesos educacionales, sino a la carencia de recursos internos (cognitivos-motivaciones-actitudinales) que disminuyen la capacidad de respuesta comportamental normativa.

De allí, que una de las causas de la deprivación sociocultural que podría explicar la presencia de comportamientos disruptivos sea las deficientes fuentes externas de estimulación, es decir, la dificultad para que un grupo de adultos en los ambientes familiar y comunitario favorezcan el proceso de socialización, se constituyan en un soporte de apoyo social y delimiten un marco normativo social (Narváez, 2019); premisa que explica porque la mayor parte de las conductas disruptivas no están asociadas a la violencia física, sino 
a la incapacidad de adoptar un marco normativo que lleve a cada estudiante a activar recursos internos de autorregulación y control de impulsos.

En cuanto a las conductas disruptivas Álvarez, Castro, González, Álvarez y Campo (2016) explican que estas se interpretan como un conglomerado de conductas inapropiadas cuya causa es la inoperancia de recursos externos (fuentes externas de estimulación) y recursos internos (fuentes de autorregulación simbólica) lo que lleva a que se tipifiquen dentro del aula comportamientos relacionados con la falta de cooperación y mala educación, desobediencia, provocación y agresión, hostilidad y abuso, impertinencia, amenazas, etc, condición pedagógica que entorpece el clima escolar con consecuencias visibles en el proceso enseñanza-aprendizaje, tal como puede evidenciarse en los resultados. Mientras, Banús (2012) identifica que estas conductas además de los factores socio-demográficos, elementos socio-pedagógicos referidos a estilos de enseñanza, aspectos personales del estudiante, características propias del profesor, factores sociales y factores familiares influyen en el proceso de aprendizaje, en especial si se trata de sujetos que están inmersos en contextos de deprivación sociocultural, donde el nivel de competencia verbal y social puede ser significativamente inferior a quienes se encuentran en ambientes comunitarios con mayores fuentes externas de estimulación.

Por otra parte, se reconocieron las conductas disruptivas asociadas a la convivencia escolar, las cuales son entendidas como una dinámica multifactorial asociada a los niveles de interacción de factores ambientales, relacionales y normativos; entre las conductas asociadas a la convivencia se encontraron las faltas de respeto, conflictos de poder y situaciones de violencia. Lo cual es corroborado, por Latorre y Taruel (2009), quienes plantean que en el aula estas conductas disruptivas fomentan el conflicto escolar alterando el clima y la sana convivencia, dado que entre los miembros de la comunidad educativa existen variedad de conductas que van desde manifestaciones de "baja intensidad, como molestar, dar respuestas inadecuadas, no respetar el turno de palabra, retar al profesor, etc., hasta agresiones físicas o verbales graves, conductas anti-sociales, absentismos, maltrato a otro compañero, y en casos extremos, a conductas derivadas de problemas psicológicos" (p.65).

Todas las conductas descritas anteriormente tienen un carácter multicausal que integran las dinámicas escolares a factores socioambientales. Entre las principales causas se encontraron los patrones sociales de violencia reproducidos en el entorno familiar y comunitario en el que está inmerso el estudiante, donde aprenden comportamientos agresivos y violentos, asimismo, se evidencia la pérdida de la autoridad moral de los adultos y 
falta de reconocimiento de la importancia de la educación y de los objetivos educativos, inversión de roles y sistemas normativos permisivos, así como las diferencias etarias entre el alumnado. Al respecto, en un estudio realizado por Urbina, Simón y Echeita (2011) encontraron que los docentes perciben que entre las diversas causas que generan este tipo de conductas disruptivas, las más representativas se compaginan con las encontradas en este estudio, entre ellas, que los padres hayan disminuido la autoridad y el control sobre sus hijos, que los alumnos no les interesa aprender ni se motivan por temas escolares y que se aburren porque en la escuela no son capaces de ofrecerles un modelo de aprendizaje motivador donde se sientan participantes activos.

En cuanto a las consecuencias se las clasificó de acuerdo a diversos factores del ambiente educativo, agentes de disrupción escolar, docentes y directivos docentes, compañeros de clase y padres de familia. Frente a ello, Jurado (2016) sugiere que entre las principales problemáticas derivadas de la disrupción se encuentra la pérdida de tiempo académico tanto para el profesor como para los alumnos, lo cual se evidencia en la cantidad de tiempo invertido por los docentes en el control y sanción de las situaciones educativas disruptivas y en la cantidad de veces que los alumnos interrumpen el proceso enseñanza-aprendizaje. De igual manera, se genera una fractura comunicacional en las aulas, se incrementa el absentismo escolar tanto por parte del alumnado como del propio profesorado y se incrementa la resistencia del cuerpo docente a emplear pedagogías activas y participativas, y en general, de cualquier tipo de iniciativa innovadora que logre minimizar el riesgo de deprivación escolar.

Al respecto, Benavides (2014) plantea que los estudiantes que crecen en lugares deprivados socioculturalmente, están en desventaja en relación con el resto de la sociedad pues estos no cuentan con las mismas oportunidades de desarrollo personal, escolar y laboral, lo que obstaculiza el normal desarrollo cognitivo, físico, emocional y social de las personas. Situaciones que afectan la motivación de los estudiantes, su atención en clases y la comprensión de lo que los docentes desean trasmitir a los estudiantes. Desde esta perspectiva, al presentar una baja motivación y un desinterés por los temas que enseñan los docentes, los estudiantes deciden ejecutar conductas que van en contra de la normatividad, reproduciendo la indisciplina y las conductas disruptivas previamente descritas, para dar cuenta de la incidencia del contexto socio-comunitario en las dinámicas escolares y de los procesos convivenciales sobre la deprivación escolar. 
Aunque para Moguel (2017) no siempre la deprivación sociocultural lleva a situaciones desadaptativas, un adolescente socialmente deprivado tendría un menor desarrollo de sus competencias sociales, situación que lo llevaría a presentar dificultades en su proceso de socialización y adaptación a marcos normativos. En ese sentido, se coloca en evidencia la relevancia del contexto sociocultural en el desarrollo de los procesos educativos, tal como lo afirman Calderón, Cruz y Rascón (2019), quienes sostienen que existe una amplia influencia del ambiente sociocultural, sobre el conjunto de comportamientos y actitudes que puedan desarrollar dentro de la escuela, siendo el contexto familiar el que más repercute en el capital cultural de los adolescentes, y uno de los factores causales de la reproducción de conductas disruptivas y oposicionistas dentro de las escuelas.

Tal argumento es corroborado por Unigarro (2013), quien en una investigación con adolescentes con dificultades de adaptación escolar e infractores de ley, identifica que gran parte de ellos provenían de ambientes comunitarios y familiares predisponentes a la deprivación sociocultural, tal es el caso de núcleos familiares fragmentados, sin roles ni límites claros y con una evidente ausencia de autoridad, o entornos barriales permeados por la violencia urbana y el microtráfico; factores que según el autor derivan en el desarrollo de comportamientos desadaptativos que dificultan su estancia en los establecimientos educativos, tal como se presenta en el acápite de resultados. Estudio que se complementa con la investigación en un grupo adolescentes entre 13 y 16 años de Martínez y Melo (2018), quienes encuentran una relación comprensiva entre las manifestaciones de la deprivación sociocultural y una situación de desventaja social observable en el proceso de aprendizaje y la convivencia de los adolescentes, donde señalan además la existencia de patrones comportamentales que agudizan la experiencia educativa, desencadenando en conductas antisociales y disruptivas.

Desde una perspectiva pedagógica, Grajales y Lizarazo (1997) proponen la existencia de relaciones teóricas entre la deprivación socio-afectiva cultural y la motivación para el aprendizaje significativo, encontrando que un adolescente en situación de deprivación sociocultural tiene conductas apáticas, desinteresadas y desganadas en sus entornos familiares, escolares y sociales; pero a diferencia de los otros estudios, los escolares tienden a desarrollar factores de disruptividad pasiva, es decir, prefieren aislarse, no participar de manera activa y no esforzarse en su desempeño. Supuesto que comparte Aarón (2017) al estudiar las circunstancias sociales que afectan los procesos escolares, donde indica que factores comunitarios como el reducido apoyo 
social y la carencia de recursos comunitarios pueden generar situaciones de deprivación socio-cultural, manifiesta en la disminuida capacidad del individuo para establecer interacciones efectivas y significativas con su entorno, lo que obstaculiza la adquisición de habilidades comunicativas y cognitivas que le permitan la promoción escolar.

Tal como lo señala Campo (2014), para quien un niño que no cuenta con las adquisiciones sociales esperadas para su edad, presenta un desarrollo emocional bajo, ocasionado por las dificultades de interacción con adultos o pares, concluyendo así la importancia de estimular y fortalecer el desarrollo personal y social en la infancia, tanto en la familia como en la escuela; estimulaciones que podrían atenuar los factores predisponentes a la deprivación sociocultural. Así pues, se hace evidente la relación interpretativa entre las condiciones del ambiente comunitario-familiar y la generación de comportamientos disruptivos en las aulas, de modo que las deficientes fuentes externas de estimulación no solo perjudican el nivel de afrontamiento a ciertos problemas vitales sino la forma como algunos estudiantes se adaptan al entorno escolar, disminuyendo el nivel de competencia verbal y social, lo que acarrea la presencia de comportamientos disruptivos y una alta probabilidad de fracaso o deserción escolar.

\section{CONCLUSIONES}

La deprivación sociocultural, entre cuyas manifestaciones se encuentra la dificultad para adoptar ambientes normativos, pocas aspiraciones escolares, comportamientos impulsivos y baja autoregulación así como dificultades en la compresión y en el dominio del lenguaje, desencadena una situación de riesgo psicosocial y de fracaso escolar en los adolescentes que presentan factores sociales y ambientales predisponentes a tal condición, derivando en deficiencias en la adaptación al ambiente escolar, lo que obstaculiza la adquisición de esquemas normativos, habilidades y competencias sociales que acarrea al desarrollo frecuente de comportamientos disruptivos.

La disrupción escolar es un fenómeno multifactorial que afecta las actividades cotidianas del salón de clase, promoviendo comportamientos asociados a la falta de respeto, los conflictos de poder y las situaciones de violencia, los cuales comúnmente retan la autoridad del docente, obligándole a emplear más tiempo en el control y el disciplinamiento de la clase; factor que minimiza el impacto del entorno educativo como fuente de estimulación externa y deriva en deprivación escolar. Entre las principales consecuencias de la disrupción escolar se encuentran el bajo rendimiento estudiantil, incumpli- 
miento de la planificación académica, baja adaptación al marco normativo escolar, incremento del ausentismo escolar, fracaso escolar e influencia negativa en el grupo. Siendo la disrupción un factor predictor de la conducta delictiva del adulto y el adolescente.

Finalmente, la disrupción escolar como fenómeno socioambiental tiene su asidero en las condiciones comunitarias, tales como ambientes barriales en precariedad económica, dinámicas relacionales conflictivas y procesos de violencia urbana y familiares, límites y roles inadecuados, pérdida de la autoridad parental y violencia intrafamiliar, todas ellas con importante incidencia en el desarrollo del estudiante, las cuales al ser predisponentes a la deprivación sociocultural se constituyen en factores de riesgo de comportamientos disruptivos. Razón por la cual, aunque existe una responsabilidad por parte del alumno como agente de conductas disruptivas, es tarea de los establecimientos educativos atender los factores de riesgo socioambiental a la disrupción para favorecer la modificación de la percepción borrosa, la impulsividad, la desorientación espacial y el déficits en la utilización de la información como funciones cognitivas deficientes que sostienen la capacidad reducida del individuo para modificar sus estructuras intelectuales, comportamentales y adaptativas al ambiente, lo que obstaculiza el alcance de objetivos escolares.

Conflicto de interés: No existe conflicto de interés en la ejecución y publicación de este artículo.

Agradecimientos: A la Institución Educativa Municipal San Juan Bosco por todo el apoyo administrativo en el desarrollo del estudio, al grupo de investigación Libres Pensadores y a los estudiantes que amablemente aceptaron participar en el estudio.

Financiamiento: Convocatoria de investigación docente Universidad de Nariño, Proyecto Estrategias de aprendizaje mediado para el desarrollo de la modificabilidad cognitiva y la disminución de la disrupción escolar en adolescentes de 13 a 17 años en situación de deprivación sociocultural de la Institución Educativa San Juan Bosco de Pasto, Colombia.

\section{REFERENCIAS}

Aarón, J. (2017). Privación cultural en estudiantes de educación por ciclos: un análisis desde los estudios cognitivos (tesis de postgrado). Universidad Distrital Francisco José de Caldas. Manizales. Colombia. http://repository.udistrital.edu. co/bitstream/11349/6696/1/FuentesLosadaJohnattanAron2017.PDF..pdf

Álvarez, M., Castro, P., González, C., Álvarez, E. y Campo, M. Á. (2016). Conductas disruptivas desde la óptica del docente: validación de una escala. Anales de Psicología, 32(3), 855-862. https://dx.doi.org/10.6018/analesps.32.3.223251 
Álvarez, C. (2008). La etnografía como modelo de investigación en educación, Gazeta de Antropología, 24(1), 1-15. http://hdl.handle.net/10481/6998

Amezcua, M. y Gálvez, A. (2002). Los modos de análisis en investigación cualitativa en salud: perspectiva crítica y reflexiones en voz alta. Revista Española de Salud Pública, 76(5), 423-436. http://scielo.isciii.es/scielo.php?script=sci_arttext\&pi$d=S 1135-57272002000500005 \&$ Ing=es\&tlng=es

Andrade Salazar, J.A., Gonzales Portillo, J. \& Calle Sandoval, D.A. (2019). Relación entre habilidades para la vida y riesgos vitales en adolescentes escolarizados de la ciudad de Armenia. Psicogente 22(42), 1-23. https://doi.org/10.17081/ psico.22.42.3490

Artigas, W. y Robles, M. (2010). Metodología de la investigación: Una discusión necesaria en Universidades Zulianas. Revista digital Universitaria, 11(11), 3-17. http://www.revista.unam.mx/vol.11/num11/art107/art107.pdf

Banús, S. (2012). Psicología infantil y juvenil. Madrid, España: Gabinete Psicológico.

Benavides, G. (2014). Competencias docentes relacionadas con la estimulación de los niveles del lenguaje verbal-oral en los estudiantes del nivel de transición de preescolar en contextos socioeconómicos de pobreza y deprivación sociocultural que asisten al Jardín de Niños Roberto Cantillano Vindas, Ipís de Goicoechea 2014 (tesis de maestria). Universidad Estatal a distancia de Costa rica, Costa rica. http:// repositorio.uned.ac.cr/reuned/bitstream/120809/1378/1/TFG\%20Gabriela\%20 \%20Benavides\%20Vega.pdf

Benavides, F., Muñoz, C. y Muñoz, G. (2013). Estado del arte sobre investigaciones educativas y pedagógicas en tres instituciones de educación superior del municipio de Pasto: conceptos y perspectivas teóricas. Revista Criterios, 20 (1), 37-51. http:// www.umariana.edu.co/ojs-editorial/index.php/criterios/article/view/366/308

Bojacá, J. (2005). El proyecto de investigación. Etnográfica en el aula marco teórico-operativo. Hallazgos, 2(3), 87-99. https://doi.org/10.15332/2422409X

Bosch, M. (2003). El reto de la escuela posmoderna. El papel de la educación en la era de la información. El Guiniguada. Revista de investigaciones y experiencias en Ciencias de la Educación, 12(1), 25-36. https://ojsspdc.ulpgc.es/ojs/index.php/ ElGuiniguada/article/view/610

Calderón, I., Cruz, O., \& Rascón, M. (2019). Aproximación al fracaso escolar de alumnado en desventaja desde el análisis crítico del discurso. Archivos Analíticos de Políticas Educativas, 27(49). http://dx.doi.org/10.14507/3538

Calvache, J. E. (2009). Investigación Cualitativa Material Técnico de Apoyo. Pasto, Colombia: Centro de Publicaciones Universidad de Nariño.

Campo Ternera, L. A. (2014). El desarrollo del autoconcepto en niños y niñas y su relación con la interacción social en la infancia. Psicogente, 17(31). https://doi. org/10.17081/psico.17.31.1470

Carrillo, E., Civis, M., Blanch, T., Longás, E. y Riera, J. (2018). Condicionantes del éxito y fracaso escolar en contextos de bajo nivel socioeconómico. Revista de Estudios y Experiencias en Educación, 2, 75-94. https://dialnet.unirioja.es/servlet/articulo? codigo $=6361387$

Castaño, C. y Quecedo, R. (2002). Introducción a la metodología de investigación cualitativa. Revista de Psicodidáctica, (14), 5-39. http://www.redalyc.org/articulo. oa?id=17501402

Cedillo, I. (2010). El Aprendizaje Mediado y las Operaciones Mentales de Comparación y Clasificación (tesis de maestría). Universidad de Cuenca, Cuenca, Ecuador. http://dspace.ucuenca.edu.ec/handle/123456789/3296 
Cladellas, R., Clariana, M. y Badia, M. (2015). Indisciplina instruccional y convencional: su predicción en el rendimiento académico. Revista Colombiana de Psicología, 24(2), 317-330. https://doi.org/10.15446/rcp.v24n2.44148

Delgado, G. (2010). Conceptos y metodología de la investigación histórica. Revista Cubana de Salud Pública, 36(1), 9-18. http://www.redalyc.org/articulo. oa?id=21416134003

Díaz, C.A (2012). La mediación, solución en la violencia escolar (tesis de maestría). Universidad Autónoma de Nuevo León, Monterrey, México. http://eprints.uanl. $\mathrm{mx} / 2731 /$

Fuica, P., Lira, J., Alvarado, K., Araneda, C., Lillo, G., Miranda, R., Tenorio, M. y Pérez, C. (2014). Habilidades Cognitivas, Contexto Rural y Urbano: Comparación de Perfiles WAIS-IV en Jóvenes. Terapia psicológica, 32(2), 143-152. http://dx.doi. org/10.4067/S0718-48082014000200007

Gordillo, E. (2013). Agrupamiento escolar y frecuencia de conductas disruptivas en estudiantes de segundo grado de educación secundaria del Callao. Educación, 22(43), 91-112. http://revistas.pucp.edu.pe/index.php/educacion/article/view/7498

Grajales, G., \& Lizarazo, F. (1997). Efectos de la deprivación afectiva-social-cultural sobre la motivación para el aprendizaje significativo (tesis de posgrado). Universidad de Antioquia. Medellín. Colombia. http://ayura.udea.edu.co:8080/jspui/ bitstream/123456789/3201/1/H0108.pdf

Hernández, R., Fernández, C. y Baptista, P. (2010). Metodología de la investigación. Distrito Federal, México: McGraw-Hill

Herrera, P. (2000). Principales factores de riesgo psicológico y social en el adolescente. Revista Cubana de Pediatría, 7(1), 39-42. http://scielo.s/d.cu/scielo.php?script $=$ sci_arttext\&pid=S0034-75312015000200005

Huertas, O. (2013). Bullying y cyberbullying: las violencias en auge entre los niños, niñas y adolescentes. Educ. Humanismo, 16 (26), 73-82. http://revistas.unisimon. edu.co/index.php/educacion/article/view/2348

Jiménez, D. (2009). Deprivación Sociocultural. Revista Temas para la Educación, (5), 1-10. https://www.feandalucia.ccoo.es/docuipdf.aspx?d=6391\&s=

Jurado, M. (2016). Propuestas de intervención ante las conductas disruptivas en la educación secundaria obligatoria. REO, 27(3), 8-25. http://www.redalyc.org/ articulo.oa?id=338250662002

Kharicha, K., lliffe, S., y Myerson, S. (2013). Why is tractable vision loss in older people being missed? Qualitative study. BMC family practice, 14(1), 99-106. http:// dx.doi.org/10.1186/1471-2296-14-99

Lastre, K., López, L. \& Alcázar, C. (2018). Relación entre apoyo familiar y el rendimiento académico en estudiantes colombianos de educación primaria. Psicogente, 21(39), 102-115. http://doi.org/10.17081/psico.21.39.2825

Latorre, A. y Teruel, J. (2009). Protocolo de actuación ante conductas disruptivas. Informacion psicológica, 95(1), 62-74. https://www.academia.edu/37119184/ Protocolo_de_actuaci\%C3\%B3n_ante_conductas_disruptivas

López, A. y Encabo, E. (2000). Intervención psicopedagógica en poblaciones con privación sociocultural: El cambio de actitudes hacia el aprendizaje de la lengua y la literatura. Revista de la Facultad de Educación de Albacete, (15), 211-220. https://dialnet.unirioja.es/servlet/articulo?codigo=2292983

Martínez, M. \& Melo, A. (2018). Factores Situacionales de privación sociocultural de un grupo de adolescentes de 13 y 16 años de la IEM San Juan Bosco-Pasto, durante el periodo B 2017 (tesis de pregrado). Universidad de Nariño. Pasto. Colombia. 
Montoya, D., Giraldo, N., Arango, L., Forgiarini, R. \& García, A. (2014). Características cognitivas, emocionales y conductuales de niños preescolares del programa buen comienzo en el noroccidente de Medellín. El Ágora U.S.B., 14(2), 637-645. https://dialnet.unirioja.es/servlet/articulo?codigo $=5372944$

Moguel, B. (2017). "iTambién existo!, creando ambientes inclusivos en alumnos de primaria con deprivación social". Perspectivas Docentes, 28(65). https://doi. org/10.19136/pd.a28n65.2580

Moreno, R. (2017). Estrategias de intervención ante la violencia verbal en estudiantes adolescentes del estado de Mérida - Venezuela. Revista Estudios y Experiencias en Educación, 16(31), 43 -53. https://doi.org/10.21703/rexe.20173143533

Narváez, J. (2019). Manifestaciones de la deprivación sociocultural y la violencia barrial en la escuela. Pasto, Editorial Universidad de Nariño, p.187

Peña, D. y Angulo, L. (2013). Conductas disruptivas presentes en estudiantes del tercer año de educación media general. Anuario del Sistema de Educación en Venezuela, 2(2), 87-110. http://erevistas.saber.ula.ve/index.php/administracioneducacional/article/view/5305

Ruiz, R., Riuró, M y Tesouro, M. (2015). Estudio del bullying en el ciclo superior de primaria. Educación XX1, 18(1), 345-368. https://doi.org/10.5944/ educxx1.18.1.12384

Serra, C. (2004). Etnografía escolar, etnografía de la educación. Revista de Educación, (334), 165-176. https://www.researchgate.net/publication/39208205_Etnografia_ escolar_etnografia_de_la_educacion

Sulbaran, A. y León, A. (2014). Estudio de las conductas disruptivas en la escuela según la percepción docente. Anuario del Sistema de Educación en Venezuela, 2(2), 35-50. http://erevistas.saber.ula.ve/index.php/administracioneducacional/ article/view/5302

Unigarro, R. (2013). Proyecto de vida de los adolescentes que presentan comportamientos conflictivos (tesis de posgrado). Universidad de Nariño. Pasto. Colombia. http://sired.udenar.edu.co/2938/1/89505.pdf

Urbina, C., Simón, C. \& Echeita, G. (2011). Concepciones de los profesores acerca de las conductas disruptivas: análisis a partir de un marco inclusivo. Infancia y Aprendizaje 34(2), 205-217. https://doi.org/10.1174/021037011795377584 